\title{
Characterization of Equine Parvovirus in Thoroughbred Breeding Horses from Germany
}

\author{
Toni Luise Meister ${ }^{1}\left(\right.$, Birthe Tegtmeyer ${ }^{2}(0)$, Yannick Brüggemann ${ }^{1}$, Harald Sieme ${ }^{3}$, \\ Karsten Feige ${ }^{3}$, Daniel Todt ${ }^{1}$, Alexander Stang ${ }^{1}$, Jessika-M.V. Cavalleri ${ }^{4, *,+}+\mathbb{C}$ and \\ Eike Steinmann $1, *,+$ \\ 1 Department of Molecular and Medical Virology, Faculty of Medicine, Ruhr-University Bochum, \\ 44801 Bochum, Germany; Toni.meister@rub.de (T.L.M.); Yannick.brueggemann@rub.de (Y.B.); \\ Daniel.todt@rub.de (D.T.); Alexander.stang@rub.de (A.S.) \\ 2 Institute for Experimental Virology, TWINCORE Centre for Experimental and Clinical Infection Research; \\ a Joint Venture between the Medical School Hannover (MHH) and the Helmholtz Centre for Infection \\ Research (HZI), 30625 Hannover, Germany; Birthe.tegtmeyer@twincore.de \\ 3 Institute of Virology; University of Veterinary Medicine Hannover, 30559 Hannover, Germany; \\ Harald.sieme@tiho-hannover.de (H.S.); Karsten.feige@tiho-hannover.de (K.F.) \\ 4 Department for Companion Animals and Horses, University of Veterinary Medicine, 1210 Vienna, Austria \\ * Correspondence: Jessika.cavalleri@vetmeduni.ac.at (J.-M.V.C.); Eike.steinmann@rub.de (E.S.); \\ Tel.: +49-234-32-23189 (E.S.) \\ + These authors contributed equally to the work.
}

Received: 4 October 2019; Accepted: 17 October 2019; Published: 18 October 2019

check for updates

\begin{abstract}
An equine parvovirus-hepatitis (EqPV-H) has been recently identified in association with equine serum hepatitis, also known as Theiler's disease. The disease was first described by Arnold Theiler in 1918 and is often observed with parenteral use of blood products in equines. However, natural ways of viral circulation and potential risk factors for transmission still remain unknown. In this study, we investigated the occurrence of EqPV-H infections in Thoroughbred horses in northern and western Germany and aimed to identify potential risk factors associated with viral infections. A total of 392 Thoroughbreds broodmares and stallions were evaluated cross-sectionally for the presence of anti-EqPV-H antibodies and EqPV-H DNA using a luciferase immunoprecipitation assay (LIPS) and a quantitative PCR, respectively. In addition, data regarding age, stud farm, breeding history, and international transportation history of each horse were collected and analysed. An occurrence of 7\% EqPV-H DNA positive and 35\% seropositive horses was observed in this study cohort. The systematic analysis of risk factors revealed that age, especially in the group of 11-15-year-old horses, and breeding history were potential risk factors that can influence the rate of EqPV-H infections. Subsequent phylogenetic analysis showed a high similarity on nucleotide level within the sequenced Thoroughbred samples. In conclusion, this study demonstrates circulating EqPV-H infections in Thoroughbred horses from central Europe and revealed age and breeding history as risk factors for EqPV-H infections.
\end{abstract}

Keywords: equine parvovirus-hepatitis; Germany; risk factors; transmission

\section{Introduction}

Equine serum hepatitis (i.e., Theiler's disease (TD)) is a serious and potentially life-threatening disease and one of the most common causes of acute hepatitis and liver failure in horses [1]. Specific treatment options are still lacking. TD was first reported in 1918 by Sir Arnold Theiler, after he observed signs of liver disease in animals vaccinated against African horse sickness with a combination of live virus and equine antiserum [2]. Similar to historical outbreaks of human posttransfusion hepatitis, 
multiple outbreaks of Theiler's disease have been observed following parenterally administered equine serum products [3-6]. An incidence between $1.4 \%-18 \%$ of fulminant hepatitis among horses receiving an equine biological product has been reported [2,7]. Given the association between prior treatment with equine serum/plasma and the appearance of Theiler's disease an etiologic role for a contaminating toxin or infectious agent has been suggested [8]. However, the exact pathogenic agent remained unknown for nearly a century.

Parvoviridae comprise a large family of non-enveloped DNA-viruses, which is currently subdivided into eight genera collectively known as parvoviruses. Members of this family have been described to infect a wide array of hosts, including humans, domestic, and wild animals [9-11]. Parvovirus infections have been associated with various severe and fatal diseases affecting the respiratory, gastrointestinal, and haematological systems and further potentially causing abortions [6,12-14]. Most recently, a novel equine Parvovirus (equine parvovirus-hepatitis virus (EqPV-H)) was isolated from serum and liver tissue of a horse that died of TD following administration of tetanus antitoxin (TAT) [15]. Administration of TAT contaminated with EqPV-H further resulted in seroconversion and acute hepatitis in experimentally infected horses, indicating that EqPV-H might be the causative agent of TD $[15,16]$. A recent study further reported a high prevalence of EqPV-H among commercial equine serum pools, indicating the necessity of careful risk assessment for medical and research applications [17]. However, despite its association with equine diseases, EqPV-H has not gained much attention of equine veterinarians and its worldwide prevalence and epidemiology remain poorly investigated.

Here, we examined the prevalence of EqPV-H among Thoroughbred breeding horses in northern and western Germany to identify potential risk factors for EqPV-H infections. A total of 392 serum samples from Thoroughbred broodmares and stallions were analysed for the presence of anti-EqPV-H antibodies, DNA, and viral sequences, respectively. Furthermore, an analysis of risk factors potentially affecting the prevalence of EqPV-H infections was performed to investigate natural routes of virus transmission.

\section{Materials and Methods}

\subsection{Serum Sample Collection}

A total of 392 serum samples from Thoroughbreds stabled on stud farms in northern and western Germany (Lower Saxony, North Rhine-Westphalia, Hamburg, Schleswig-Holstein) representing more than $25 \%$ of all registered breeding horses at The German Thoroughbred Studbook Authority (Cologne) were collected and processed between 2012 and 2015 [18]. All samples were then stored at $-80^{\circ} \mathrm{C}$ until further analysis regarding the presence of EqPV-H.

\subsection{Detection of $E q P V-H D N A$}

Viral DNA was extracted with a viral DNA Kit from Qiagen (Cat. No. 1048147, Hilden, Germany) according to the manufacturer's recommendations. DNA samples were stored at $-20^{\circ} \mathrm{C}$ until further analysis. A probe-based quantitative polymerase chain reaction (qPCR) was used with primers and probe designed and provided by Dr. Amit Kapoor as described previously [15]. A serial dilution of a plasmid containing the EqPV-H VP1 sequence was generated as standard row for the quantification of EqPV-H within the samples tested. qPCR measurements were performed using the LightCycler 480 real-time PCR system (Roche, Mannheim, Germany). 


\subsection{Detection of Anti-EqPV-H Antibodies}

Samples were analysed for the presence of anti-EqPV-H-VP1 antibodies using the previously described luciferase immunoprecipitation system (LIPS) [19-21]. The EqPV-H-LIPS antigen VP1 was produced as described by Divers et al. [15]. Following the LIPS assay, relative light units (RLU) were determined using a plate luminometer (LB 960 XS3; Berthold, Bad Wildbad, Germany). To calculate sensitivity the mean RLU plus three standard deviations (SD) of an EqPV-H negative horse serum was defined as a cut-off limit. A potential cross-reactivity between the LIPS and other related parvoviruses could not be excluded.

\subsection{Data Collection and Study Design}

Three different groups regarding the state of EqPV-H infection were distinguished: Seropositive and EqPV-H DNA positive $\left(\mathrm{DNA}^{+} / \mathrm{AB}^{+}\right)$, seronegative and EqPV-H DNA negative $\left(\mathrm{DNA}^{-} / \mathrm{AB}^{-}\right)$, and seropositive and EqPV-H DNA negative $\left(\mathrm{DNA}^{-} / \mathrm{AB}^{+}\right)$. Information regarding gender, age, state of reproduction, breeding, and international transportation history of the study population were received earlier from the Association for breeding and racing of Thoroughbreds (Cologne, Germany). Furthermore, the established criteria were subdivided into different groups. Age groups of similar size were created: 3-6-year-old horses $(n=78), 7-10$-year-old horses $(n=113), 11-15$-year-old horses $(n=129)$, and 16-29-year-old horses $(n=72)$. Similarly, horses were assigned to groups regarding the breeding history (0-4 breeding years) and the stock size of the stud farms (1-9 horses, 10-39 horses and $>40$ horses). Additional information about the transportation history was sorted according to the target country (France, Great Britain, Ireland, Germany, etc.). The data were determined by the time of sample collection between 2012-2015.

\subsection{Sequencing and Phylogeny}

For sequence analysis, a PCR (I) was designed within the NS1 of EqPV-H [17]. PCR was performed using the expand high fidelity PCR system (Roche Diagnostics, Basel, Switzerland) as described before [17]. PCR products were visualised on a $\%$ agarose gel, excised, and purified using a Monarch ${ }^{\circledR}$ DNA gel extraction kit (New England Biolabs, Ipswich, Massachusetts, United States). Purified products were then sent for Sanger sequencing using the applicable PCR primers. Highlighter plot analysis [22] displaying nucleotide exchanges in EqPV NS1 in the screened cohort compared to a previously published strain from Europe (MK792434). Labelling of nucleotides according to IUPAC code. Length of bar scale of neighbour-joining tree indicating number of nucleotide exchanges. The input multiple sequence alignment was created with Mega X.

\section{Results}

\subsection{Frequent Occurrence of EqPV-H among German Thoroughbreds}

We first investigated the frequency of EqPV-H infections among Thoroughbreds from the north and west of Germany. A total of 392 serum samples from Thoroughbreds originating from Lower Saxony and North Rhine-Westphalia were collected during an annual fertility monitoring and tested for the presence of anti-EqPV-H-VP1 antibodies and EqPV-H DNA, respectively (Figure 1A). A total of 28 samples were tested positive for EqPV-H DNA (7.14\%) via qPCR. We further evaluated the samples for the presence of anti-VP1-antibodies using a previously described LIPS [17]. An elevated serum titre of anti-EqPV-H-VP1 antibodies above the detection limit was present in $136(34.69 \%)$ horses. Based on these findings the individual horses were assigned to the following groups: $\mathrm{DNA}^{-} / \mathrm{AB}^{-} ; \mathrm{DNA}^{-} / \mathrm{AB}^{+}$ and $\mathrm{DNA}^{+} / \mathrm{AB}^{+}$(Figure 1B). Interestingly, no sample was positive for EqPV-H DNA only, indicating no acute EqPV-H infections within the examined cohort. 
A

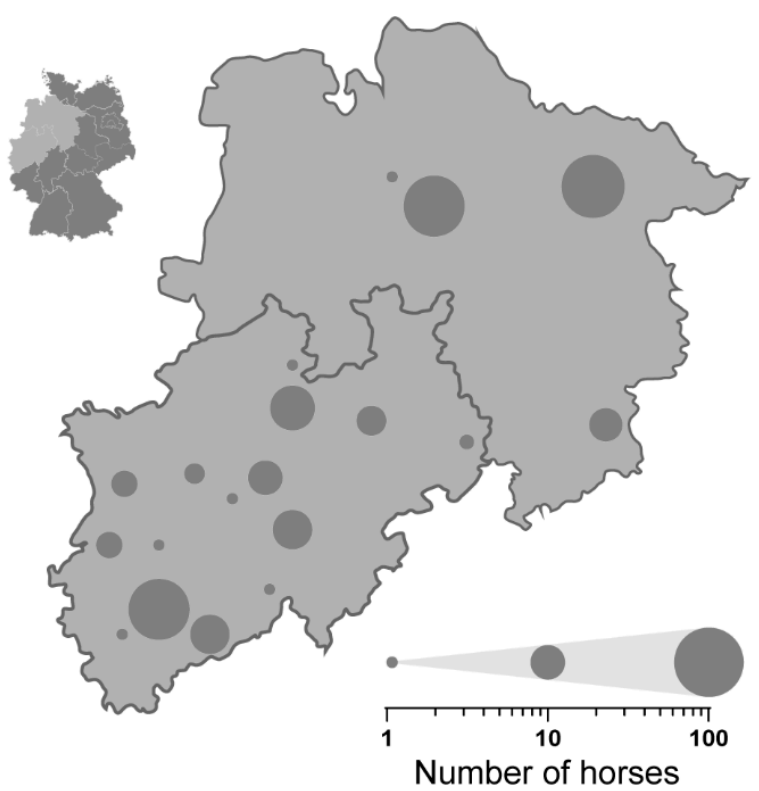

B

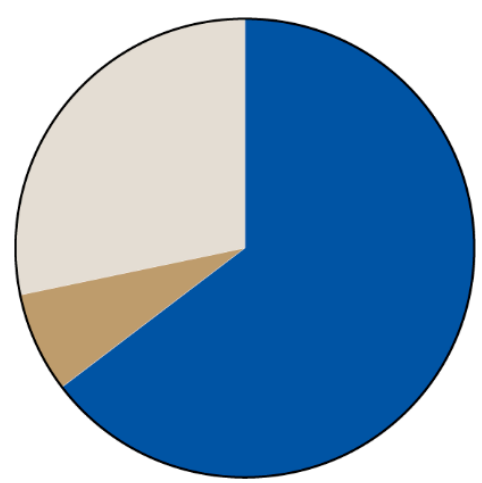

Total $=392$

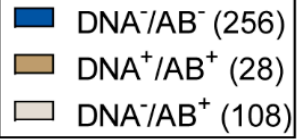

Figure 1. (A) Map of Germany highlighting the location of sampling (insert upper left). Serum samples were collected from 392 Thoroughbreds in North Rhine-Westphalia and Lower Saxony. Grey circles highlight the sample location and circle size is scaled to relative the number of examined horses (see legend lower right). (B) Serum samples were analysed for the presence of anti-equine parvovirus-hepatitis (anti-EqPV-H) VP1 AB and EqPV-H DNA performing luciferase immunoprecipitation system (LIPS) and $\mathrm{qPCR}$, respectively. Individual horses were assigned to three different groups: Seronegative and EqPV-H DNA negative ( $\mathrm{DNA}^{-} / \mathrm{AB}^{-} ; 65.3 \%$ ), seropositive and EqPV-H DNA negative ( $\mathrm{DNA}^{-} / \mathrm{AB}^{+} ; 27.55 \%$ ), and seropositive and EqPV-H DNA positive $\left(\mathrm{DNA}^{+} / \mathrm{AB}^{+} ; 7.14 \%\right)$.

\subsection{Viral Characteristics of EqPV-H-Positive Horses}

Next, we characterized the distribution of viral loads in the study cohort showing three high-titre samples with copy numbers above $5 \times 10^{4} \mathrm{DNA}$ copies/mL (Figure 2A). For the anti-EqPV-H-VP1 antibodies, titres up to a 600-fold increase over the limit of detection were observed (Figure 2B). Interestingly, $\mathrm{DNA}^{+} / \mathrm{AB}^{+}$samples showed significantly higher titres of anti-VP1-antibodies as compared to the $\mathrm{DNA}^{-} / \mathrm{AB}^{+}$horses (Figure $2 \mathrm{C}$ ). The $28 \mathrm{EqPV}-\mathrm{H}$ positive samples were further confirmed in a gel electrophoresis of the qPCR products (Figure 2D). The highest viral loads were detected for samples 13, $14,15,20$, and 23, respectively (Figure 2E). The highest anti-VP1-antibody levels were observed for samples 10, 15, 16, 18, and 23 (Figure 2F). 
A

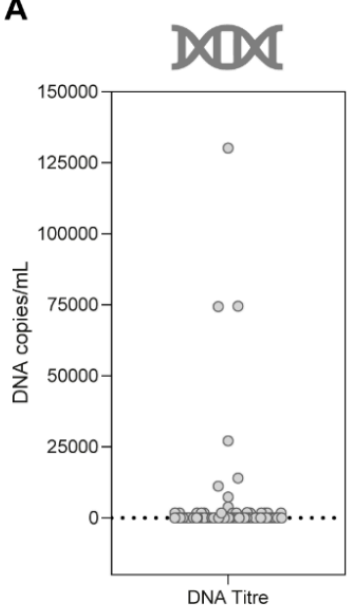

B

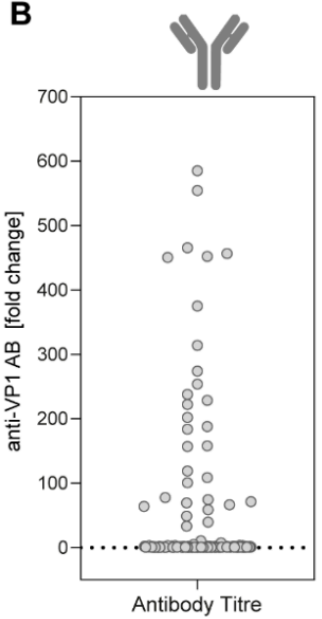

C

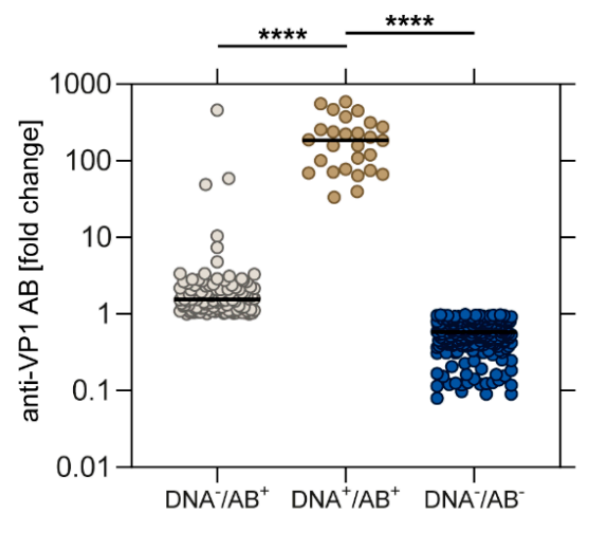

D
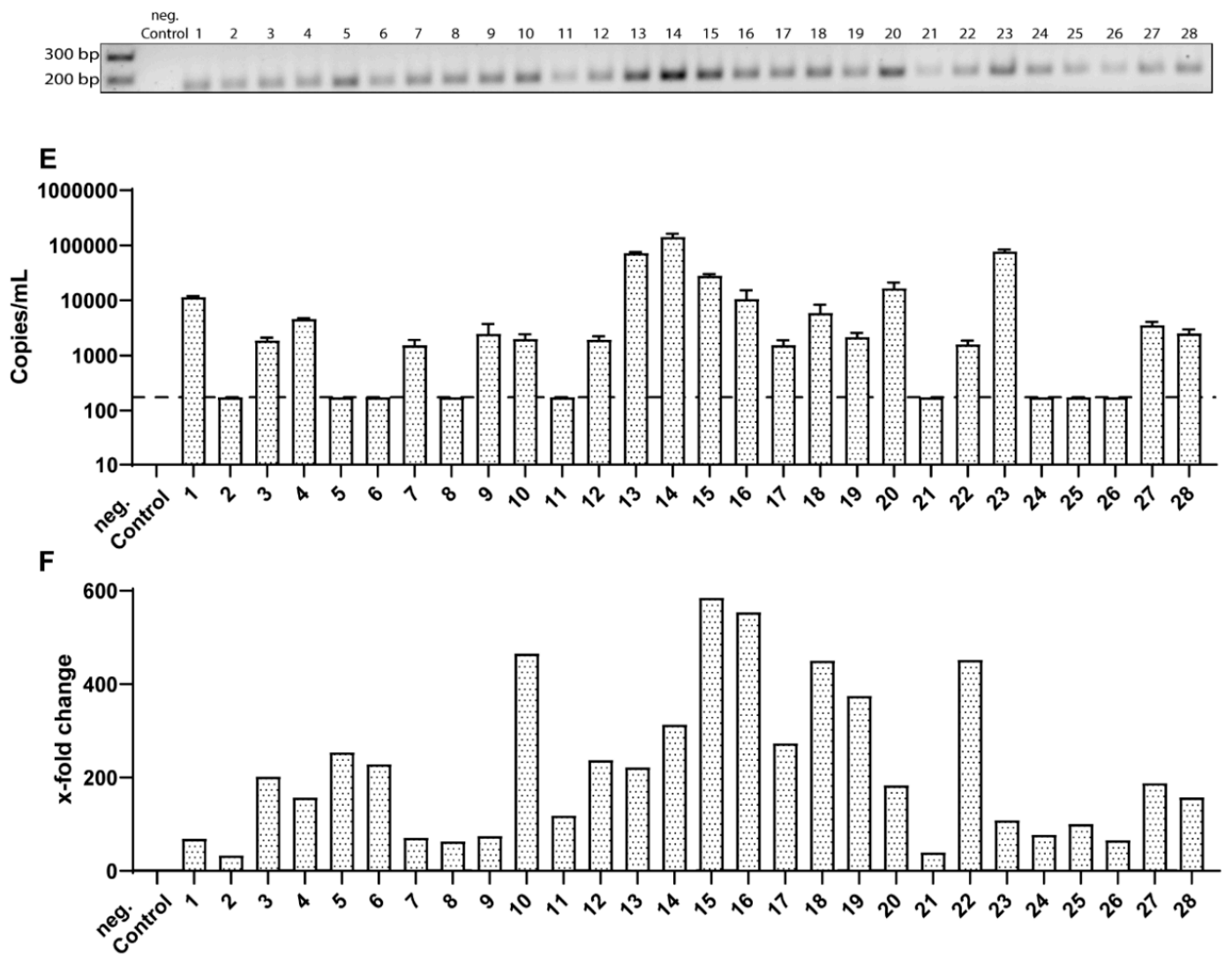

Figure 2. (A) Viral loads of EqPV-H (DNA copies/mL) were determined via qPCR $(n=392)$. Horses with viral load of $>175$ copies/mL were considered EqPV-H DNA positive. (B) EqPV-H VP1 antibodies were detected using a LIPS assay and the relative increase of RLU compared to an EqPV-H-negative control sample was calculated $(n=392)$. (C) Comparison of relative EqPV-H VP1 antibody levels between different groups: (Median values; $\mathrm{DNA}^{-} / \mathrm{AB}^{-}, n=256 ; \mathrm{DNA}^{-} / \mathrm{AB}^{+}, n=108 ; \mathrm{DNA}^{+} / \mathrm{AB}^{+}$, $n=28)$. Statistical significance was determined using a one-way ANOVA with Dunnett's post-hoc test. ( $\left.{ }^{* * * *} p<0.0001\right)$. (D) Agarose gel electrophoresis of the qPCR product from DNA positive serum samples $(n=28)$ and an EqPV-H-negative control sample. (E) Viral loads of EqPV-H as determined by qPCR are displayed in DNA copies/mL. The dotted line indicates the limit of detection (mean +/$\mathrm{SD}, n=3)$. (F) Evaluation of serum titres for anti-EqPV-H antibodies in EqPV-H DNA positive serum samples determined by LIPS. RLU was normalised to the cut-off limit and is displayed as $x$-fold change. 


\subsection{Age and Breeding History are Potential Risk Factors of EqPV-H Infection in Thoroughbreds}

We next performed an analysis using data on age, breeding history, stock size of the stud farm, and transportation to a foreign country to determine factors, which might be involved in promoting EqPV-H and would identify natural routes of transmission. All results of the descriptive analysis are shown in Figure 3, with detailed information provided in Table 1. By analysing the impact of the age on EqPV-H infection, it became apparent that with increasing age, especially between 11-15 years, the proportion of EqPV-H DNA positive and seropositive horses $\left[\mathrm{DNA}^{+} / \mathrm{AB}^{+}\right]$was elevated (Figure $3 \mathrm{~A}$ ). In the group of 3-6-year-old horses approximately $14 \%$ were tested DNA positive and seropositive as compared to $43 \%$ for the group of $11-15$ and $25 \%$ for the group of 16-29-year-old horses (Figure 3A). Likewise, a slight increase in the fraction of seropositive horses was observed upon increasing breeding history (Figure 3B). Regarding the classification of the stud farm based on their stock size, no difference between the different groups could be noted (Figure 3C). More horses were recruited from farms with more than 40 horses (Table 1). Most of the sampled and analysed horses stayed in Germany and other countries including Great Britain $(n=27)$, Ireland $(n=33)$, and France $(n=26)$. Importantly, travelling history to another country was not associated with an increased risk for EqPV-H infection (Figure 3D and Table 1).
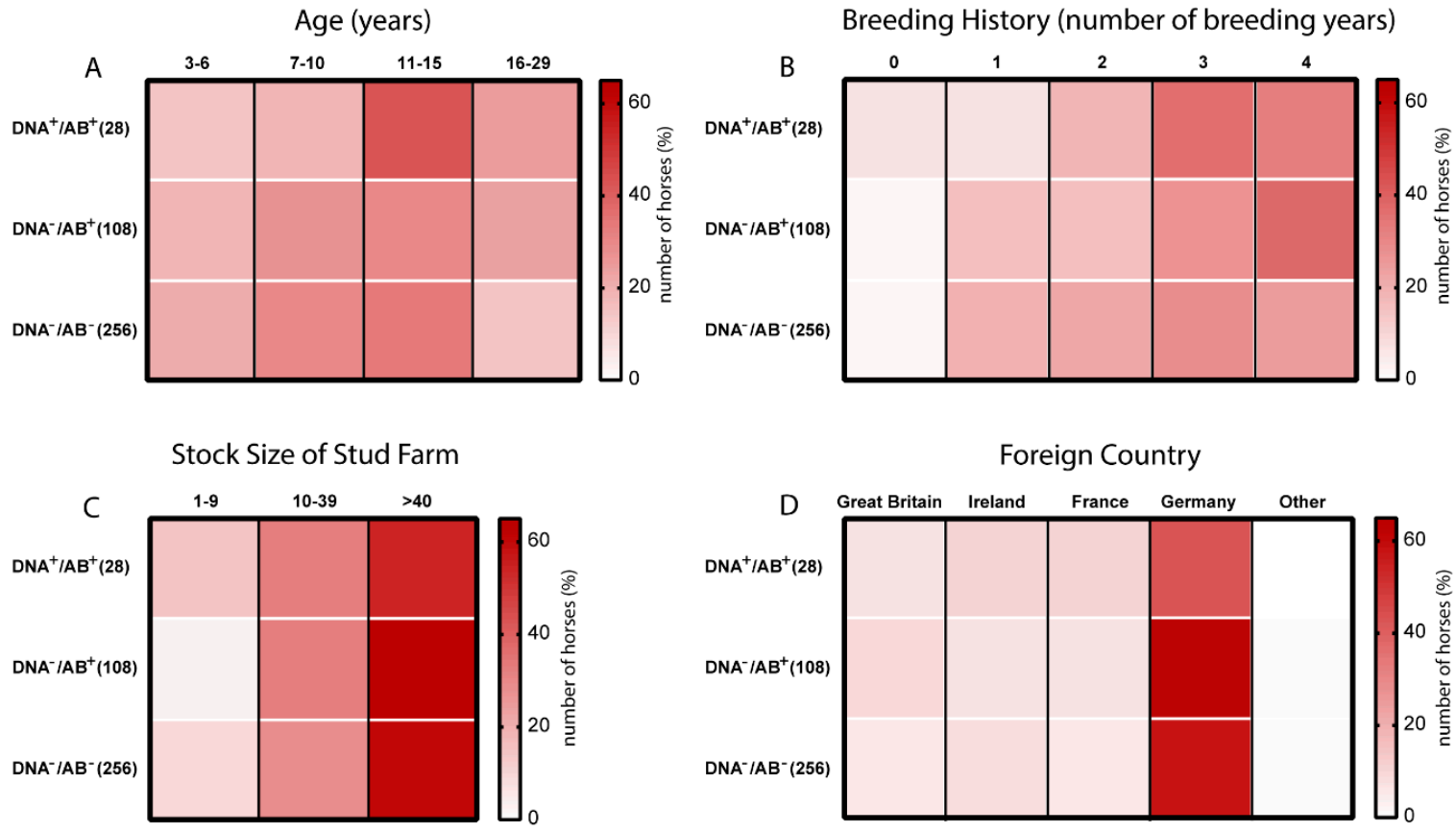

Figure 3. Heatmaps displaying the occurrence of anti-EqPV-H VP1 AB and EqPV-H DNA with regard to potential risk factors. The three groups based on the EqPV-H infection status were further classified based on (A) age, (B) breeding history, (C) stock size of the stud farm, and (D) foreign country. The colouring indicates the number of horses (in \%) classified in the specific category. Each row adds up to $100 \%$. 
Table 1. General information regarding gender, age, breeding history, stock size, travel background, and EqHV-coinfection of the equine serum samples collected from Thoroughbreds in North Rhine-Westphalia and Lower Saxony.

\begin{tabular}{|c|c|c|c|c|c|c|}
\hline \multirow{3}{*}{ Variables $(n)$} & \multicolumn{6}{|c|}{ State of EqPV-H Infection } \\
\hline & \multicolumn{2}{|c|}{$\mathrm{DNA}^{-} / \mathrm{AB}^{+}(108)$} & \multicolumn{2}{|c|}{$\mathrm{DNA}^{+} / \mathrm{AB}^{+}(27) *$} & \multicolumn{2}{|c|}{$\mathrm{DNA}^{-} / \mathrm{AB}^{-}(256)$} \\
\hline & $n$ & $\%$ & $n$ & $\%$ & $n$ & $\%$ \\
\hline \multicolumn{7}{|l|}{ GENDER ** } \\
\hline Mare (380) & 106 & 98.15 & 26 & 92.86 & 248 & 96.88 \\
\hline Stallion (10) & 2 & 1.85 & & & 8 & 3.12 \\
\hline \multicolumn{7}{|l|}{ AGE } \\
\hline $3-6$ years $(78)$ & 20 & 18.52 & 4 & 14.29 & 54 & 21.09 \\
\hline $7-10$ years $(113)$ & 30 & 27.78 & 5 & 17.86 & 78 & 30.47 \\
\hline $11-15$ years (129) & 32 & 29.6 & 12 & 42.86 & 85 & 33.20 \\
\hline $16-29$ years $(72)$ & 26 & 24.07 & 7 & 25 & 39 & 15.23 \\
\hline \multicolumn{7}{|c|}{ NUMBER OF BREEDING YEARS } \\
\hline Not covered (9) & 2 & 1.85 & 2 & 7.14 & 5 & 1.95 \\
\hline 1 breeding year (68) & 17 & 15.74 & 2 & 7.14 & 49 & 19.14 \\
\hline 2 breeding year $(80)$ & 17 & 15.74 & 5 & 17.86 & 58 & 22.66 \\
\hline 3 breeding year (113) & 29 & 26.85 & 10 & 35.71 & 74 & 28.91 \\
\hline 4 breeding year (112) & 41 & 37.96 & 9 & 32.14 & 62 & 24.22 \\
\hline \multicolumn{7}{|l|}{ STOCK SIZE } \\
\hline 1-9 horses (34) & 4 & 3.70 & 4 & 14.29 & 26 & 10.16 \\
\hline 10-39 horses (119) & 35 & 32.41 & 9 & 32.14 & 75 & 29.30 \\
\hline$>40$ horses $(239)$ & 69 & 63.89 & 15 & 53.57 & 155 & 60.55 \\
\hline \multicolumn{7}{|l|}{ FOREIGN COUNTRY ** } \\
\hline Great Britain (27) & 10 & 9.26 & 2 & 7.14 & 15 & 5.86 \\
\hline Ireland (33) & 8 & 7.41 & 3 & 10.71 & 22 & 8.59 \\
\hline France (26) & 8 & 7.41 & 3 & 10.71 & 15 & 5.856 \\
\hline Germany (227) & 67 & 62.04 & 12 & 42.86 & 148 & 57.81 \\
\hline Other (4) & 1 & 0.93 & 0 & 0 & 3 & 1.172 \\
\hline \multicolumn{7}{|l|}{ EqHV-COINFECTION ** } \\
\hline $\mathrm{RNA}^{-} / \mathrm{AB}^{+}(196)$ & 60 & 55.57 & 12 & 44.45 & 124 & 48.44 \\
\hline $\mathrm{RNA}^{+} / \mathrm{AB}^{+}(41)$ & 8 & 7.41 & 4 & 14.82 & 29 & 11.33 \\
\hline $\mathrm{RNA}^{-} / \mathrm{AB}^{-}(152)$ & 40 & 37.04 & 10 & 37.04 & 102 & 39.84 \\
\hline
\end{tabular}

${ }^{*}$ Data regarding potential risk factors could only be obtained for 27 of the $28 \mathrm{DNA}^{+} / \mathrm{AB}^{+}$horses. ${ }^{* *}$ Data regarding gender, foreign country, and EqHV-coinfection could not be obtained for all the 392 horses.

In a recent study, we observed a frequent occurrence of equine hepacivirus (EqHV) infections among the same cohort of Thoroughbreds with approximately $62 \%$ seropositive horses for EqHV antibodies [18]. We surveyed whether an EqPV-H co-infection potentially favours an EqHV infection. However, a similar prevalence of approximately $60 \%$ seropositivity for EqHV was observed among all groups of horses, indicating that an EqHV infection does not predispose individual horses for an EqPV-H infection (Table 1 and Figure S1). Overall, we observed an age dependent state of EqPV-H infection and our results further suggest a slightly higher prevalence among horses with an extended breeding history. In contrast, no correlation between the stock size of the stud farm and transportation history to a foreign country could be observed indicating that neither acts as a predisposing factor for an EqPV-H infection.

\subsection{Sequence and Phylogenetic Analysis of EqPV-H Detected in Thoroughbreds}

We next performed a molecular characterization of the EqPV-H DNA positive samples. A PCR (I) was designed to amplify a region within the NS1 gene of EqPV-H and the obtained amplicons were subjected to conventional Sanger sequencing. The obtained sequences were submitted to the GenBank database with the accession numbers indicated in Table 2. Due to very low viral loads sequencing 
reactions could not be successfully performed for all samples (20/28 sequences for PCRI). The obtained sequences were highly similar to a previously described EqPV-H sequence (Figure 4). As indicated by the highlighter plot and the length of the tree branches, a high grade of genomic conservation is found within the analysed cohort. Sequence identity with the previously described European strain is ranging from $95 \%$ to $98 \%$ (Figure 4). In accordance with previous findings these results indicate a high degree of conservation and genomic stability between the world-wide circulating strains and low genetic variability of the EqPV-H strain.

Table 2. The newly identified specimens were submitted to the National Centre for Biotechnology Information (NCBI) and were assigned to the following accession numbers. The sample ID refers to Figure 2D-F.

\begin{tabular}{ccc}
\hline Sample ID & EqPV Sequence Name & NCBI Accession Number \\
\hline 1 & EqPV-H/VB1_518-1104nt & MN184860 \\
3 & EqPV-H/VB3_518-1104nt & MN184861 \\
4 & EqPV-H/VB4_518-1104nt & MN184862 \\
10 & EqPV-H/VB10_518-1104nt & MN184863 \\
11 & EqPV-H/VB11_518-1104nt & MN184864 \\
17 & EqPV-H/VB17_518-1104nt & MN184865 \\
5 & EqPV-H/VB5_518-1104nt & MN184866 \\
19 & EqPV-H/VB19_518-1104nt & MN184867 \\
20 & EqPV-H/VB20_518-1104nt & MN184868 \\
23 & EqPV-H/VB23_518-1104nt & MN184869 \\
12 & EqPV-H/VB12_518-1104nt & MN184870 \\
14 & EqPV-H/VB14_518-1104nt & MN184871 \\
24 & EqPV-H/VB24_518-1104nt & MN184873 \\
25 & EqPV-H/VB25_518-1104nt & MN184874 \\
27 & EqPV-H/VB27_518-1104nt & MN184875 \\
15 & EqPV-H/VB15_518-1104nt & \\
\hline
\end{tabular}

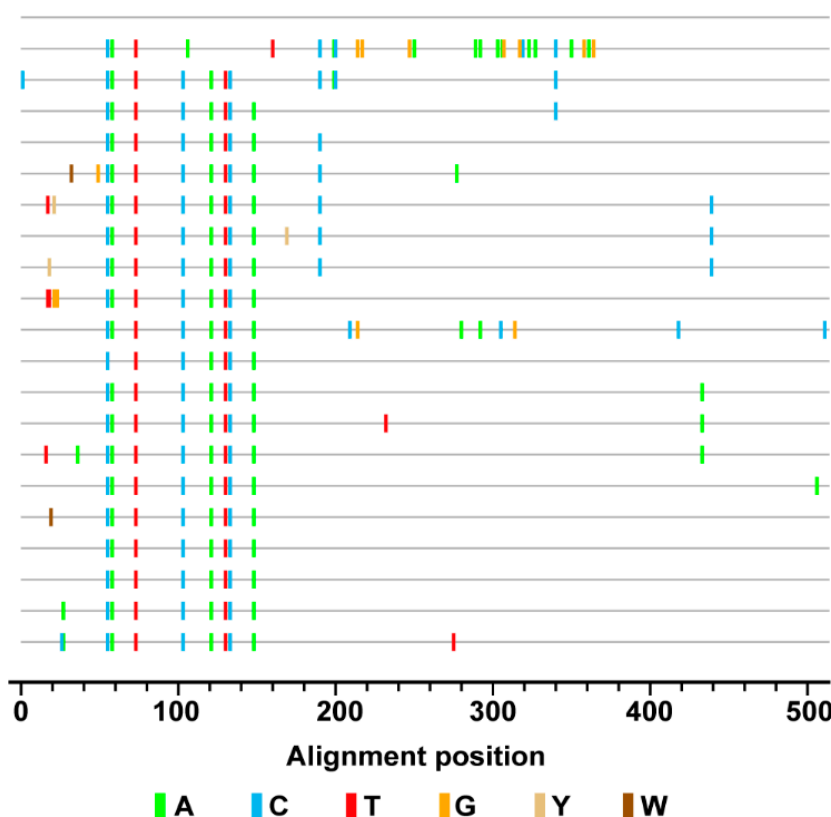

Figure 4. Highlighter plot displaying nucleotide exchanges in EqPV NS1 in the screened cohort compared to a previously published strain from Europe (MK792434). Labelling of nucleotides according to the International Union of Pure and Applied Chemistry (IUPAC) code. Length of bar scale of neighbour-joining tree indicating number of nucleotide exchanges. The input multiple sequence alignment was created with Mega X.

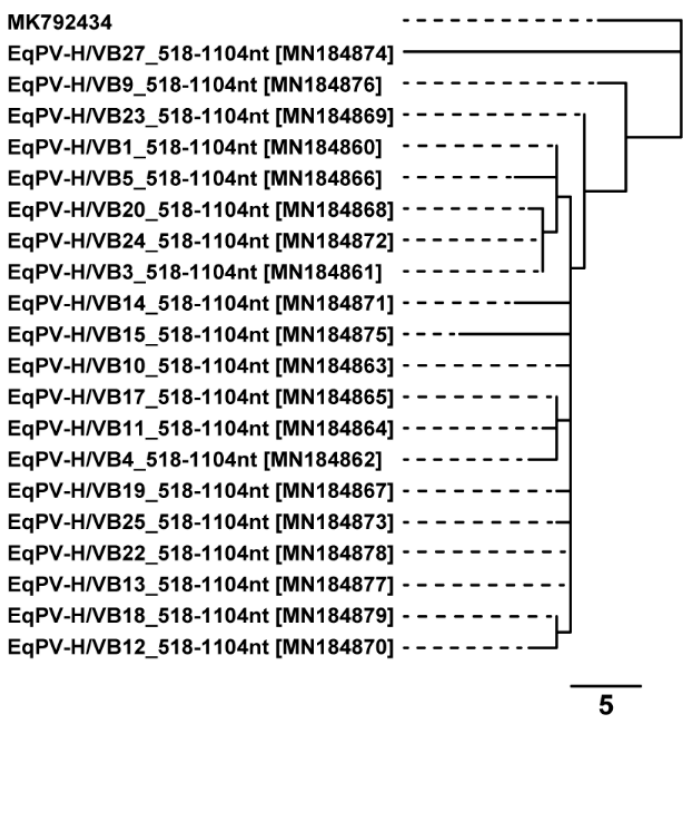

\section{MK792434}

EPV-HIVB9 518-1104nt [MN184876]

EqPV-H/VB1_518-1104nt [MN184860] . . . . . . -

EqPV-H/VB5 518-1104nt [MN184866] - - - - -

EqPV-HIVB24_518-1104nt [MN184872] - - - - -

EqPV-H/VB3 518-1104nt [MN184861]

Q

EqPV-H/VB10_518-1104nt [MN184863] - - - - - - -

VB17_518-1104nt [MN184865] - -

EqPV-H/VB4_518-1104nt [MN184862]

EqPV-H/VB19_518-1104nt [MN184867] - - - - - - -

qPV-H/VB25 518-1104nt [MN184873] - - - - - -

EqPV-H/VB13 518-1104nt [MN184877] - - - - - -

EqPV-H/VB18_518-1104nt [MN184879] - - - - - - -

EqPV-H/VB12 518-1104nt [MN184870] - . . . . . . 


\section{Discussion}

In this cross-sectional study, we examined the prevalence of EqPV-H among almost 400 Thoroughbreds in northern and western Germany representing the first analysed European country. Based on the information from the German Association for breeding and racing of Thoroughbreds (Cologne), a total of 1450 brood mares and about 80 stallions were registered as breeding horses in 2014. Thus, we investigated about a quarter of the actively breeding Thoroughbreds in Germany and the results can be considered representative for the region. We observed a frequent occurrence of EqPV-H infections with $34.69 \%$ seropositive and $7.14 \%$ viraemic horses. The phylogenetic analysis demonstrated a high level of conservation in NS1 sequences in comparison with the world-wide circulating strains implying low levels of genetic variability. A detection of EqPV-H DNA with concurrent absence of anti-EqPV-H-VP1 antibodies was not observed for any sample, indicating no acute infection at the time of sampling. Of note, a cross-reaction in the LIPS assay with other potential parvoviruses in the horse samples cannot fully be excluded. Previously, in the first report describing the identification of EqPV-H a PCR prevalence in serum of 13\% (13/100) was reported in the USA [15], and in China the average prevalence was $11.9 \%$ (17/143) in racehorses [11]. Further prevalence studies with larger cohorts from various continents are required for a more detailed epidemiology of EqPV-H.

We also performed a descriptive comparison between potential risk factors affecting EqPV-H prevalence. Iatrogenic transmission of EqPV-H has been demonstrated by Divers et al. through the administration of equine origin blood products [15], which should be confirmed ideally with a clonal viral EqPV-H inoculum in future studies. Of note, the virus can also be transmitted to horses that did not have such treatments [8]. The relative number of viraemic and seropositive horses in our analysis was increased with advanced age, especially between 11 and 15 years of age, but also in the group of 16-29-year-old horses compared to $\mathrm{DNA}^{-} / \mathrm{AB}^{-}$and $\mathrm{DNA}^{-} / \mathrm{AB}^{+}$horses. Possible reasons can be persistent humoral immunity or high risk of re-infection with longer living times. Due to the study design, horses younger than three years were not included. Therefore, it is impossible to draw a conclusion whether young foals in which the immune system is still maturing are at an increased risk of infection. Stage of reproduction was additionally accompanied by slightly higher fraction of EqPV-H infections. However, given that an extended breeding history mostly involves an advanced age of the examined horse this observation might be due to an indirect correlation. Neither stock size of the stud farm, transportation history to a foreign country, or an EqHV infection could be identified as a predisposing factor.

Of note, our previous investigation of the same study cohort revealed that younger Thoroughbreds transported abroad were at a higher risk to get infected with EqHV than horses at the same age staying in Germany. It is described that transportation of horses [23-25] and a changing environment as well as disruption of established social groups [26] can lead to a stress-induced immunosuppression, which could favour viral transmission. This risk factor seems therefore not to be highly involved in the susceptibility of horses to EqPV-H infections. Furthermore, equine hepacivirus co-infection was not a determinant for increased EqPV-H infections. Recently, a high rate of co-infections of these two equine viruses was also described in the prevalence report by Lu et al. from China [11]. As an additional way of viral spreading, we also investigated the potential of vertical transmission of EqPV-H in a single case. Postnatal serum samples were taken from a foal after delivery from an EqPV-H DNA positive and anti-VP1-positive mare (Figure S2). Transfer of EqPV-H-specific antibodies to the foal via colostrum could be observed, but no viremia was detected. The specific antibody transfer was expected, since the colostrum represents the important route of antibody transfer in neonatal foals, whereas the placental barrier hampers intrauterine transfer of macromolecules such as immunoglobulins from the mare to the foetus [27].

In conclusion, these data revealed that Thoroughbreds from central Europe have a frequent occurrence of anti-VP1 antibodies and EqPV-H DNA suggesting circulating EqPV-H infections in the horse population, endemic herds, or persistent shedding. In addition, age and multiple breeding were identified as potential risk factors for EqPV-H transmission. 
Supplementary Materials: The following are available online at http:/www.mdpi.com/1999-4915/11/10/965/s1, Figure S1: Correlation between EqPV-H and EqHV status of thoroughbreds. The EqPV-H status is displayed on the abscissa with the EqHV status shown in four shades of grey. Data for EqHV RNA and seroprevalence were obtained during a previous study [18]; Figure S2: Postnatal serum samples from a foal and an EqPV-H-positive mare were evaluated for the presence of EqPV-H VP1 antibodies and DNA at the indicated times using a luciferase immunoprecipitation (LIPS) assay and the relative increase of RLU compared to an EqPV-H-negative control sample was calculated.

Author Contributions: Conceptualization, J.-M.V.C. and E.S.; Methodology, T.L.M., B.T., H.S., D.T., and A.S.; Formal analysis, Y.B., H.S., K.F., D.T., A.S., J.-M.V.C., and E.S.; Investigation, T.L.M. and B.T.; Resources, H.S., K.F., J.-M.V.C., and E.S.; Data curation, T.L.M. and A.S.; Writing-original draft preparation, T.L.M., Y.B., and E.S.; Writing-review and editing, T.L.M., B.T., Y.B., H.S., K.F., D.T., A.S., J.-M.V.C., and E.S.; Visualization, T.L.M. and Y.B.; Supervision, J.-M.V.C. and E.S.; Project administration, J.-M.V.C. and E.S.

Funding: This research received no external funding.

Acknowledgments: We are grateful to Amit Kapoor for providing us with the qPCR protocol and also thank Peter D. Burbelo (NIH, Maryland, USA) for providing the Renilla-luciferase-VP1 fusion plasmid. We would like to thank all members of the Department of Molecular and Medical Virology at the Ruhr-University Bochum and especially Rosemarie Bohr, Regina Bütermann, Monika Kopytkowski, Klaus Sure, and Ute Wiegmann-Misiek for technical support. We additionally acknowledge support by the DFG Open Access Publication Funds of the Ruhr-Universität Bochum.

Conflicts of Interest: The authors declare no conflict of interest.

\section{References}

1. Sturgeon, B. Theiler's disease. Vet. Rec. 2017, 180, 14-15. [CrossRef] [PubMed]

2. Theiler, A. Acute Liver-Atrophy and Parenchymatous Hepatitis in Horses. In 5th and 6th Repts. of the Director of Veterinary Research; Dept. of Agriculture, Union of South Africa: Pretoria, South Africa, 1918; pp. 7-164.

3. Panciera, R.J. Serum hepatitis in the horse. J. Am. Vet. Med. Assoc. 1969, 155, 408-410. [PubMed]

4. Guglick, M.A.; MacAllister, C.G.; Ely, R.W.; Edwards, W.C. Hepatic disease associated with administration of tetanus antitoxin in eight horses. J. Am. Vet. Med. Assoc. 1995, 206, 1737-1740. [PubMed]

5. Aleman, M.; Nieto, J.E.; Carr, E.A.; Carlson, G.P. Serum Hepatitis Associated with Commercial Plasma Transfusion in Horses. J. Vet. Intern. Med. 2005, 19, 120-122. [CrossRef] [PubMed]

6. Chandriani, S.; Skewes-Cox, P.; Zhong, W.; Ganem, D.E.; Divers, T.J.; van Blaricum, A.J.; Tennant, B.C.; Kistler, A.L. Identification of a previously undescribed divergent virus from the Flaviviridae family in an outbreak of equine serum hepatitis. Proc. Natl. Acad. Sci. USA 2013, 110, E1407-E1415. [CrossRef]

7. Tomlinson, J.E.; Tennant, B.C.; Struzyna, A.; Mrad, D.; Browne, N.; Whelchel, D.; Johnson, P.J.; Jamieson, C.; Löhr, C.V.; Bildfell, R.; et al. Viral testing of 10 cases of Theiler's disease and 37 in-contact horses in the absence of equine biologic product administration, A prospective study (2014-2018). J. Vet. Intern. Med. 2019, 33, 258-265. [CrossRef]

8. Tomlinson, J.E.; van de Walle, G.R.; Divers, T.J. What Do We Know About Hepatitis Viruses in Horses? The Veterinary clinics of North America. Equine Pract. 2019, 35, 351-362. [CrossRef]

9. Cotmore, S.F.; Agbandje-McKenna, M.; Chiorini, J.A.; Mukha, D.V.; Pintel, D.J.; Qiu, J.; Soderlund-Venermo, M.; Tattersall, P.; Tijssen, P.; Gatherer, D.; et al. The family Parvoviridae. Arch. Virol. 2014, 159, 1239-1247. [CrossRef]

10. Palinski, R.M.; Mitra, N.; Hause, B.M. Discovery of a novel Parvovirinae virus, porcine parvovirus 7, by metagenomic sequencing of porcine rectal swabs. Virus Genes 2016, 52, 564-567. [CrossRef]

11. Lu, G.; Sun, L.; Ou, J.; Xu, H.; Wu, L.; Li, S. Identification and genetic characterization of a novel parvovirus associated with serum hepatitis in horses in China. Emerg. Microbes Infect. 2018, 7, 170. [CrossRef]

12. Wong, F.C.; Spearman, J.G.; Smolenski, M.A.; Loewen, P.C. Equine Parvovirus: Initial Isolation and Partial Characterization. Can. J. Comp. Med. 1985, 49, 50-54. [PubMed]

13. Li, L.; Giannitti, F.; Low, J.; Keyes, C.; Ullmann, L.S.; Deng, X.; Aleman, M.; Pesavento, P.A.; Pusterla, N.; Delwart, E. Exploring the virome of diseased horses. J. Gen. Virol. 2015, 96, 2721-2733. [CrossRef] [PubMed]

14. Tomlinson, J.E.; Kapoor, A.; Kumar, A.; Tennant, B.C.; Laverack, M.A.; Beard, L.; Delph, K.; Davis, E.; Schott Ii, H.; Lascola, K.; et al. Viral testing of 18 consecutive cases of equine serum hepatitis, A prospective study (2014-2018). J. Vet. Intern. Med. 2019, 33, 251-257. [CrossRef] [PubMed] 
15. Divers, T.J.; Tennant, B.C.; Kumar, A.; McDonough, S.; Cullen, J.; Bhuva, N.; Jain, K.; Chauhan, L.S.; Scheel, T.K.H.; Lipkin, W.I.; et al. New Parvovirus Associated with Serum Hepatitis in Horses after Inoculation of Common Biological Product. Emerg. Infect. Dis. 2018, 24, 303-310. [CrossRef]

16. Divers, T.J.; Tomlinson, J.E. Theiler's disease. Equine Vet. Educ. 2019, 19, 120. [CrossRef]

17. Meister, T.L.; Tegtmeyer, B.; Postel, A.; Cavalleri, J.-M.V.; Todt, D.; Stang, A.; Steinmann, E. Equine Parvovirus-Hepatitis Frequently Detectable in Commercial Equine Serum Pools. Viruses 2019, 11, 461. [CrossRef]

18. Reichert, C.; Campe, A.; Walter, S.; Pfaender, S.; Welsch, K.; Ruddat, I.; Sieme, H.; Feige, K.; Steinmann, E.; Cavalleri, J.M.V. Frequent occurrence of nonprimate hepacivirus infections in Thoroughbred breeding horses-A cross-sectional study for the occurrence of infections and potential risk factors. Vet. Microbiol. 2017, 203, 315-322. [CrossRef]

19. Burbelo, P.D.; Ching, K.H.; Klimavicz, C.M.; Iadarola, M.J. Antibody profiling by Luciferase Immunoprecipitation Systems (LIPS). J. Vis. Exp. JoVE 2009, e1549. [CrossRef]

20. Burbelo, P.D.; Dubovi, E.J.; Simmonds, P.; Medina, J.L.; Henriquez, J.A.; Mishra, N.; Wagner, J.; Tokarz, R.; Cullen, J.M.; Iadarola, M.J.; et al. Serology-enabled discovery of genetically diverse hepaciviruses in a new host. J. Virol. 2012, 86, 6171-6178. [CrossRef]

21. Pfaender, S.; Cavalleri, J.M.V.; Walter, S.; Doerrbecker, J.; Campana, B.; Brown, R.J.P.; Burbelo, P.D.; Postel, A.; Hahn, K.; Anggakusuma Riebesehl, N.; et al. Clinical course of infection and viral tissue tropism of hepatitis C virus-like nonprimate hepaciviruses in horses. Hepatol. (Baltim. Md.) 2015, 61, 447-459. [CrossRef]

22. Keele, B.F.; Giorgi, E.E.; Salazar-Gonzalez, J.F.; Decker, J.M.; Pham, K.T.; Salazar, M.G.; Sun, C.; Grayson, T.; Wang, S.; Li, H.; et al. Identification and characterization of transmitted and early founder virus envelopes in primary HIV-1 infection. Proc. Natl. Acad. Sci. USA 2008, 105, 7552-7557. [CrossRef] [PubMed]

23. Grandin, T. Assessment of stress during handling and transport. J. Anim. Sci. 1997, 75, 249-257. [CrossRef] [PubMed]

24. Smith, B.L.; Jones, J.H.; Hornof, W.J.; Miles, J.A.; Longworth, K.E.; Willits, N.H. Effects of road transport on indices of stress in horses. Equine Vet. J. 1996, 28, 446-454. [CrossRef] [PubMed]

25. Schmidt, A.; Biau, S.; Möstl, E.; Becker-Birck, M.; Morillon, B.; Aurich, J.; Faure, J.-M.; Aurich, C. Changes in cortisol release and heart rate variability in sport horses during long-distance road transport. Domest. Anim. Endocrinol. 2010, 38, 179-189. [CrossRef]

26. Alexander, S.L.; Irvine, C.H. The effect of social stress on adrenal axis activity in horses: The importance of monitoring corticosteroid-binding globulin capacity. J. Endocrinol. 1998, 157, 425-432. [CrossRef]

27. Chucri, T.M.; Monteiro, J.M.; Lima, A.R.; Salvadori, M.L.B.; Kfoury, J.R.; Miglino, M.A. A review of immune transfer by the placenta. J. Reprod. Immunol. 2010, 87, 14-20. [CrossRef] 